\title{
ParameTerized Sub-Systems of HVAC Piping System
}

\author{
Tomáš Kalina, Vladislav Kemka \& Zdeněk Chval
}
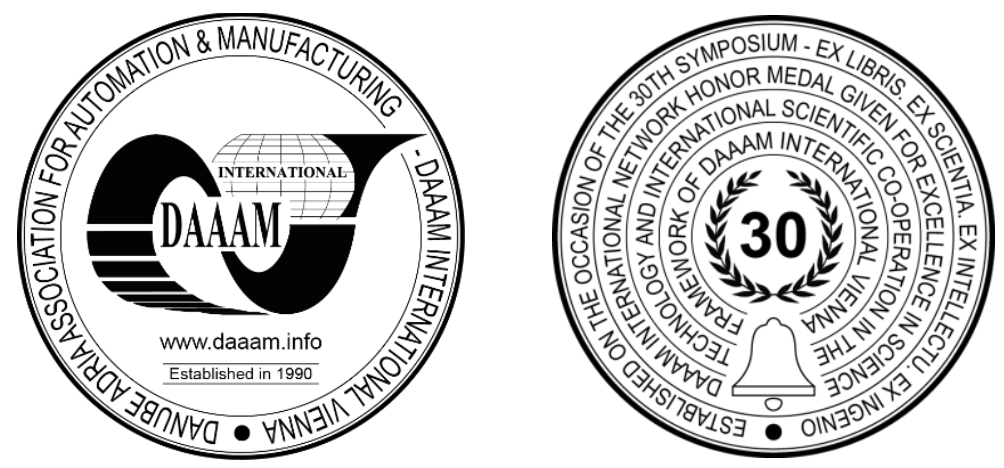

This Publication has to be referred as: Kalina, T[omas]; Kemka, V[ladislav] \& Chval, Z[denek] (2019). Parameterized Sub-systems of HVAC Piping System, Proceedings of the 30th DAAAM International Symposium, pp.0615-0621, B. Katalinic (Ed.), Published by DAAAM International, ISBN 978-3-902734-22-8, ISSN 1726-9679, Vienna, Austria DOI: $10.2507 / 30$ th.daaam.proceedings.084

\begin{abstract}
The goal of this paper is to introduce the proposed parameterized sub-systems of HVAC piping system $(\mathrm{HVAC}=$ heating, ventilation, air conditioning). Parameterized 3D models of piping sub-systems and supports of piping system were created. Using control parameters we can control not only the size of pipes and supports, but with logical parameters $(0 / 1)$ and conditions, we can change the sub-system type, or show and hide individual parts of the sub-system. This spectrum of parameterized models enables quick and efficient design of HVAC piping system.
\end{abstract}

Keywords: parameterized 3D model; HVAC; piping system; optimization.

\section{Introduction}

At our workplace we deal with design and optimization of HVAC piping system (HVAC = heating, ventilation, air conditioning). Most often, these are pipelines for combustion plants and heating plants [7]. In such plants, these are mainly large diameter pipes (most often in the range of $300-1000 \mathrm{~mm}$ ). Pipes of such large dimensions must be securely fastened to the structure. We deal with design, optimization and structural [1] and temperature [2], [3], [4], [10] numerical simulations of piping systems and supports. The design takes into account the resulting functionality and efficiency, manufacturability, assembly and safety during production and operation. In case of a failure [8], [9] we also deal with the analysis of the problem, such as in the publication [1], where excessive load and failure of supports and piping are solved.

The motivation to create parameterized sub-models was born because identical or similar elements are often repeated on the piping system. Simple and fast modifiable models can save a great deal of time.

In the CAD system - Siemens NX 12.0 parameterized 3D models and drawings are created. Finite element models and simulations are created also in Siemens NX 12.0. 


\section{Piping system and supports of piping system}

Figure 1 shows a part of the piping system (in this case it is specifically the recirculation circuit of the combustion plant). The piping system consists of purchased pipes, pipes made of sheets and supports.

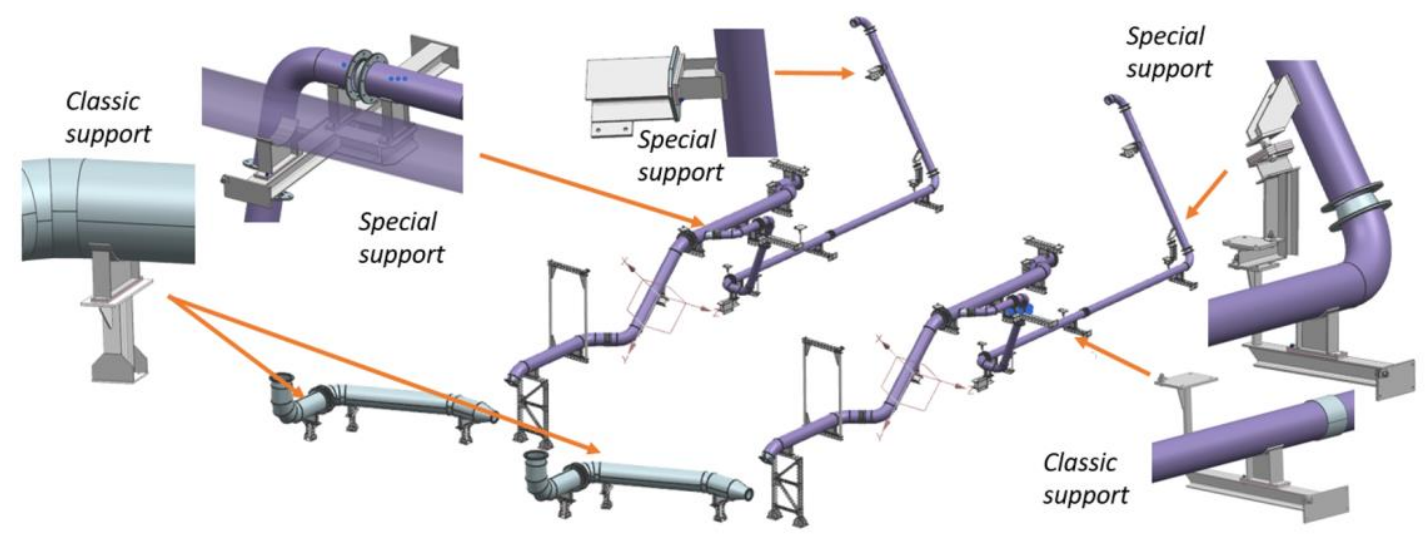

Fig. 1. Sample recirculation circuit combustion plants

\section{Parameterized piping system}

Fully parametric 3D models of pipes, pipe elbows and pipe transition parts have been created to optimize the pipeline design process. Thanks to fully parametric 3D models, simply by rewriting several parameter values in the table, the 3D model of the given segment is completely changed, which are then assembled into a pipe line.

Figure 2 below shows 3D models of pipes and elbows with their control parameters. In addition to the pipe dimensions, the radius and angle of the knee and the cutting of the ends at the desired angles can be controlled by means of the parameters. The 3D model is designed so that the tubes can be directly unfolded on a sheet metal flat pattern (for laser) to create a drawing of the part with the basic dimensions.
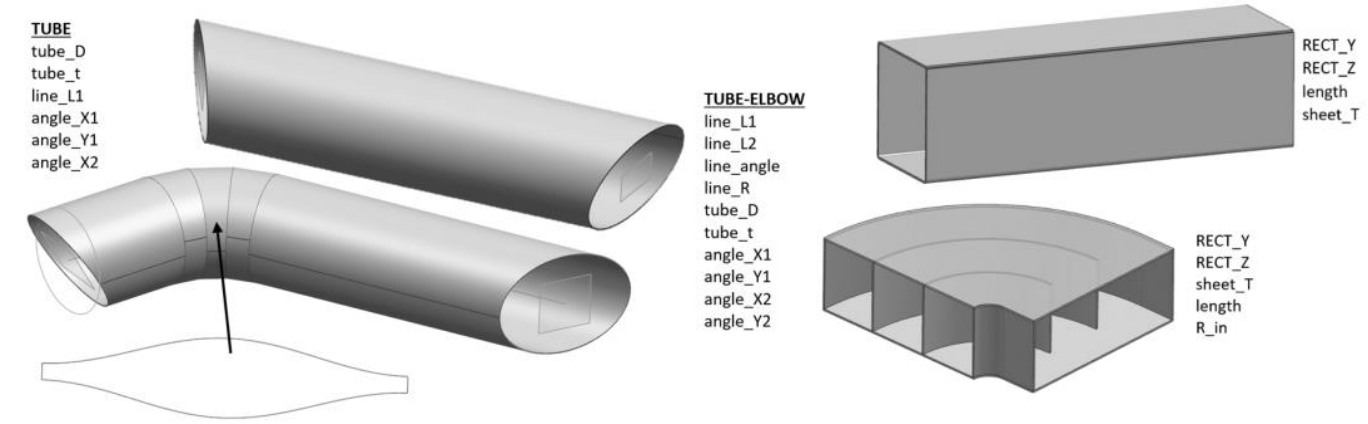

Fig. 2. Parametrized model of a tube and an elbow and control parameters and demonstration of flat-pattern of one sheet

Different transition elements are used when changing the pipe cross-section. Examples of transition elements are shown in Fig. 3 below. The control parameters are the dimensions of both ends, the total length and the offset of the centers of both ends relative to each other. All these transitions are made from one piece of sheet metal and the flat pattern is automatically generated.
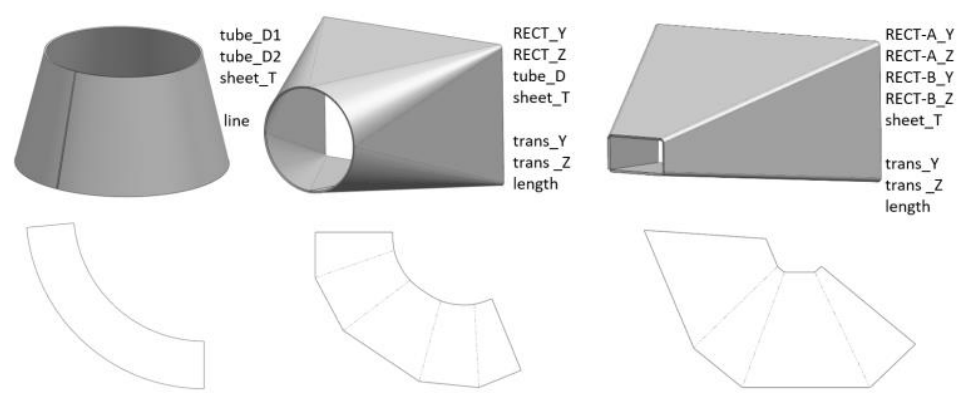

Fig. 3. Transition elements 
Fans, preheaters [4], [5], compensators and control valves are often part of the HVAC piping system. All these elements are fixed into the pipeline by means of flanges. Fig. 4 shows parametric models of flanges and deflectors that are part of compensators. The flanges are controlled by only one (circular flanges) or two (rectangular flanges) parameters that control the pipe dimension (internal flange dimension). All remaining dimensions depend on the pipe diameter. The flanges have a uniform thickness of $10 \mathrm{~mm}$. The number and pitch of holes is calculated from the condition that the hole pitch must be between 100 and $150 \mathrm{~mm}$ and the number of holes for bolts is always even.

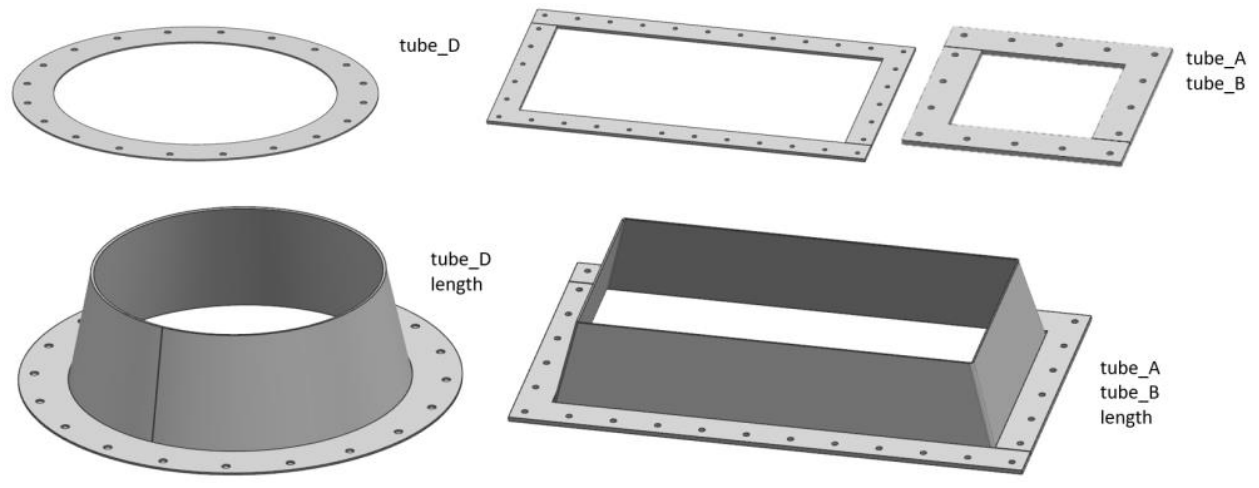

Fig. 4. Flanges and deflectors

\section{Parameterized supports of piping system}

Most supports are made of sheets and profiles. Therefore, scalable models of HEA, IPE and UPE profiles were created. Figure 5 shows an example of a scalable HEA profile. It is possible to choose from several standardized dimensions, but also to control the parameters of holes and cut the ends of the profile. These profiles serve as the basic building element for further support.

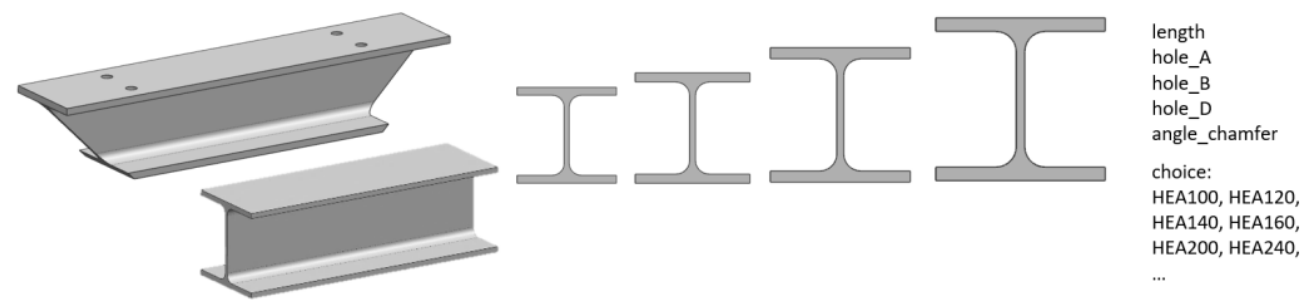

Fig. 5. Scalable model of HEA profile

Figure 6 shows a 3D model of a classic two-point hinge, which is very versatile. In addition to the dimensions, it is possible to switch between the symmetrical and asymmetrical design of the mounting foot and the tubes independently. Individual components can also be suppressed/displayed. All supports are mounted to the supporting structure using lindapters [11], which is basically a clamping connection.

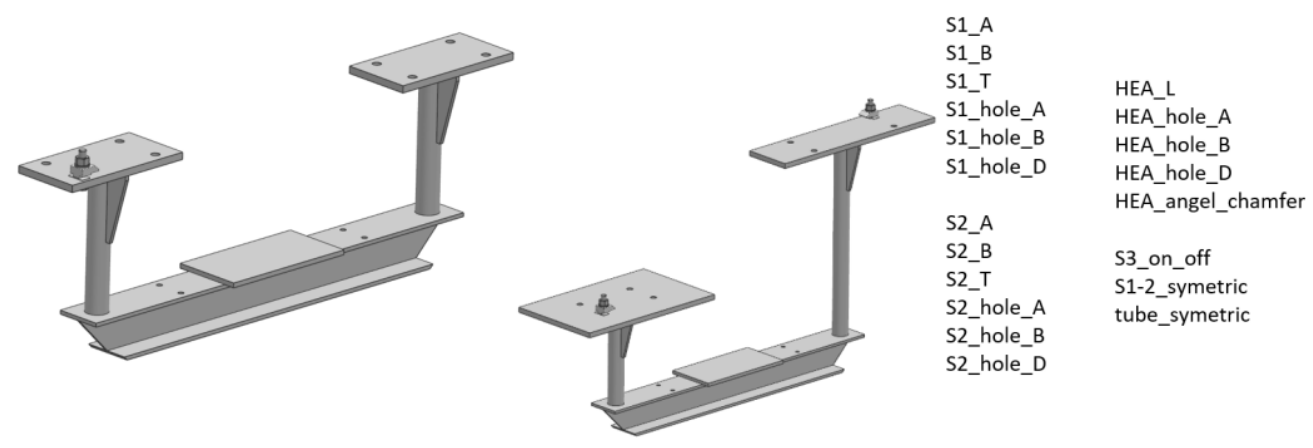

Fig. 6. Two-point hinge

Another frequently occurring support is the beam on two feet (Fig. 7). Here, again, it is possible to set the symmetrical/asymmetrical design, to suppress components, but also to control the position of the feet whether they are on the side or top surface of the profile with one logical parameter. 


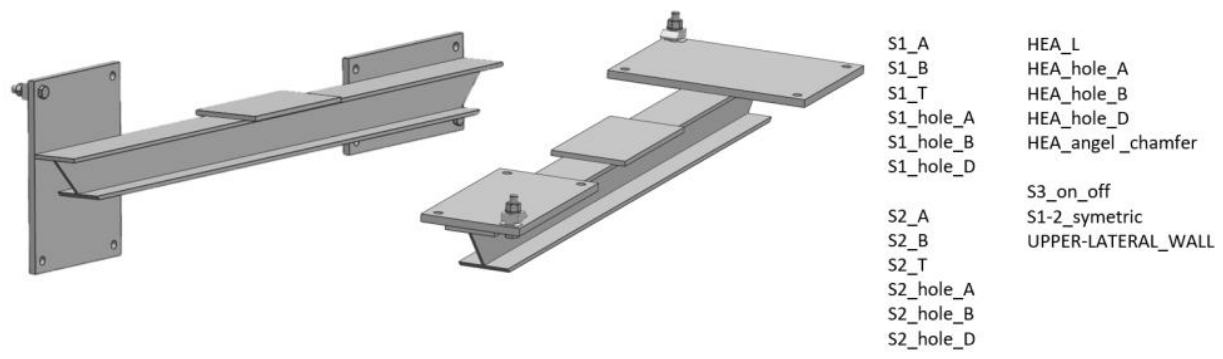

Fig. 7. Beam on two feet

Similarly, a 3D model of additional support is constructed in Fig. 8, wherein the feet are located on the faces of the profiles. This type of support has the same options as the previous model, and it is also possible to rotate the profile to the feet at any angle.
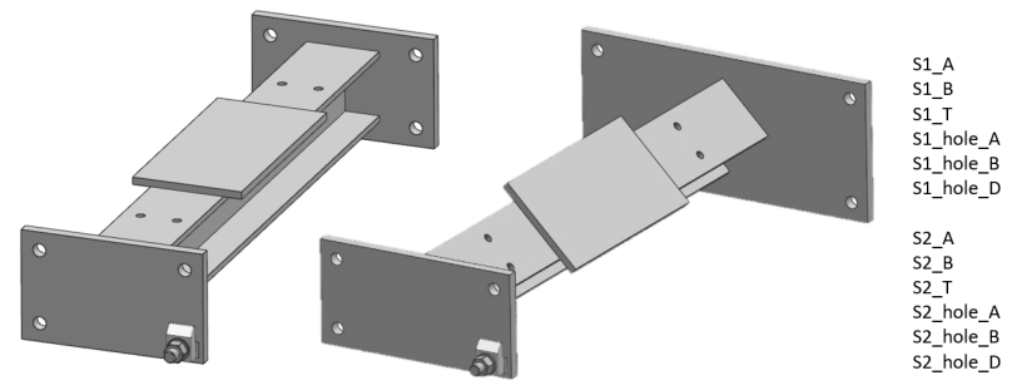

HEA_hole_D

HEA_angel_chamfer

S3_on_off

S1-2_symetric

HEA-SHEET_angle

Fig. 8. Beam on two feet - front feet

For anchoring to the floor or foundations, the single-leg support (Fig. 9) is often used, where it is possible to change the dimensions and to choose between sliding or fixing bonding.
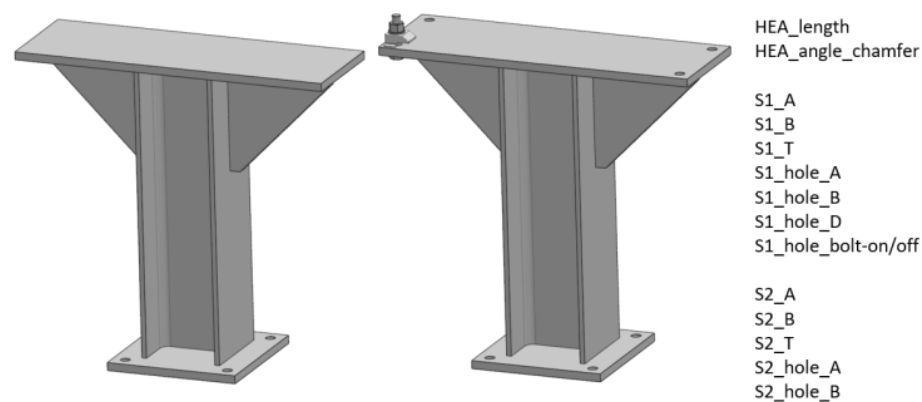

Fig. 9. Simple leg - floor mounting

On the above-mentioned type of support it is possible to place the bottom support (Fig. 10), where it is possible to choose between fixed and sliding bonding.
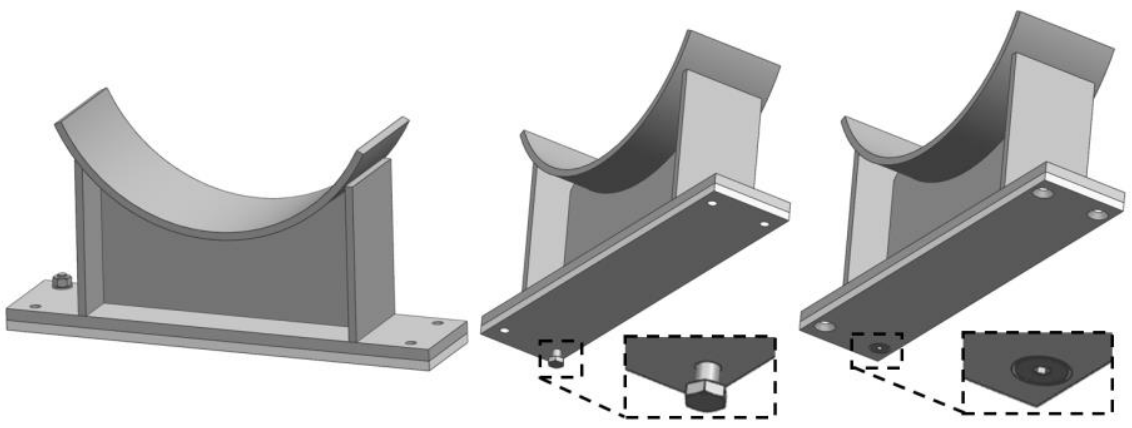

tube_D

height_to_axis

Base_A

Base_B

Base_hole_A

Base_hole_B

Base_hole_D

FIX_FREE-POINT

Fig. 10. Bottom support - fixed (b) and sliding version (c) 
When mounting into the floor or foundation, where the pipe is at a higher height, the higher leg is shown, as shown in Fig. 11 below.

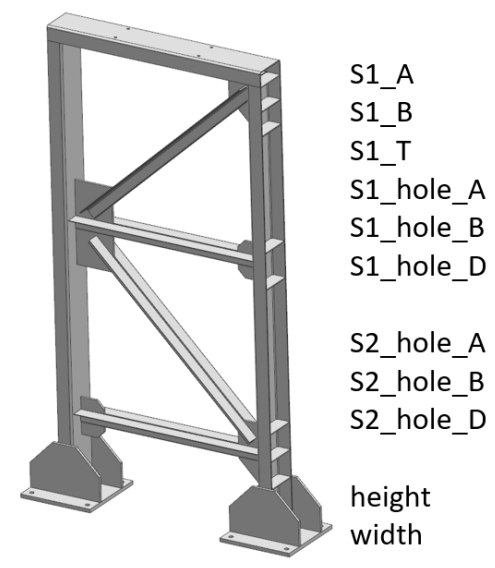

Fig. 11. Higher leg

An insulating layer must always be placed between the pipe and the supporting structure. Usually it is a plastic plate that is clamped between two steel plates. The insulating plate is made of PTFE for hot pipes and PA [6] for cold pipes. An example of an insulating plate is shown in Fig. 10. In Fig. 10 b) (Fix point) serves only as thermal insulation. Fig. c) (sliding point) serves as thermal insulation and as a sliding material.

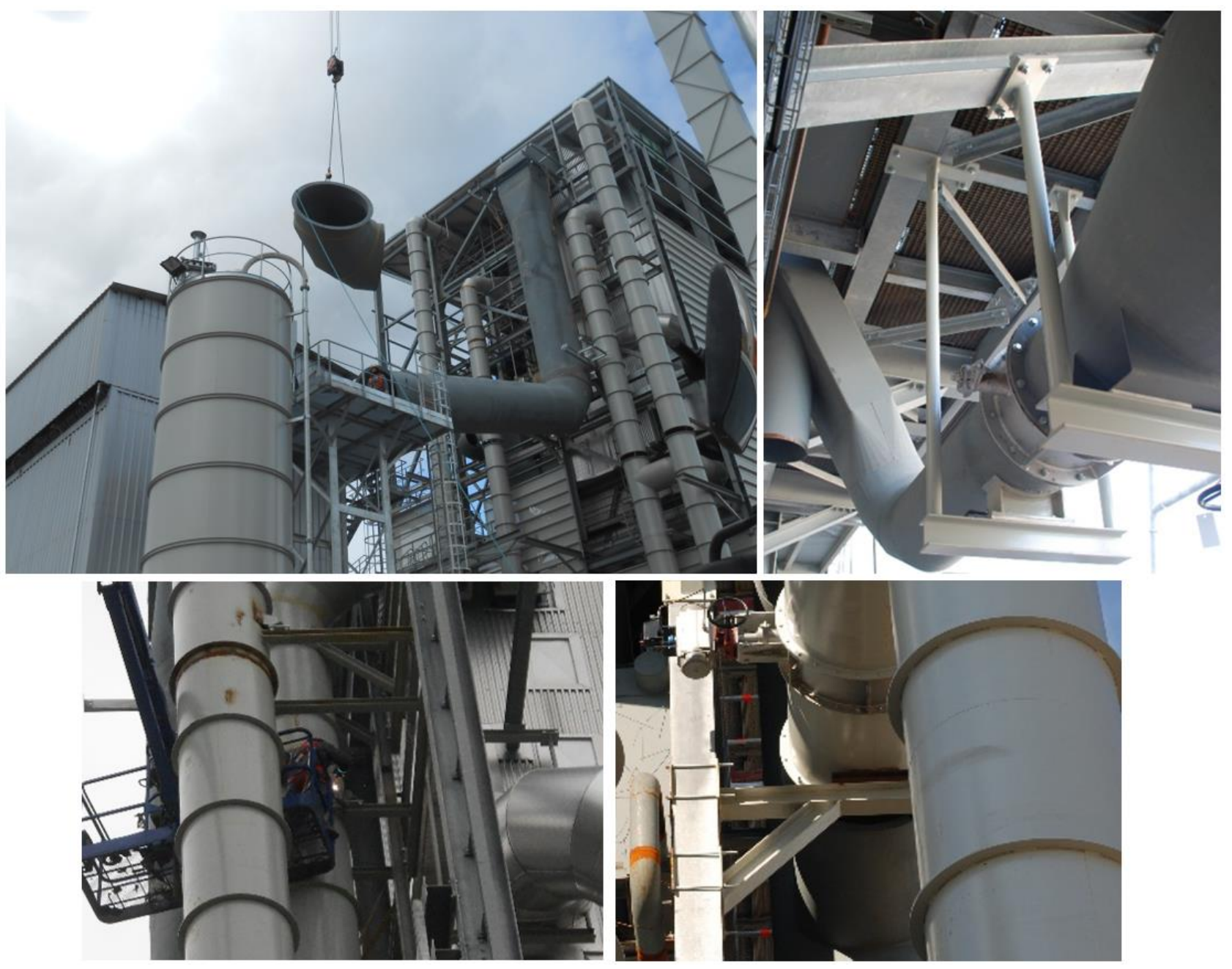

Fig. 12. Photo-documentation from real incinerator construction - detail on selected piping parts and supports 


\section{Flow simulation and optimization}

Simulations and possible optimization of the air flow in the ducts are carried out during the design of piping system. Fig. 13 shows an example of air temperature simulation. There is a preheater in the extended rectangular part. Before the pre-heater is a cold medium and behind pre-heater is a medium with a temperature exceeding $100^{\circ} \mathrm{C}$.

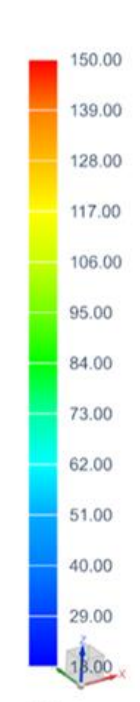

$\left[{ }^{\circ} \mathrm{C}\right]$

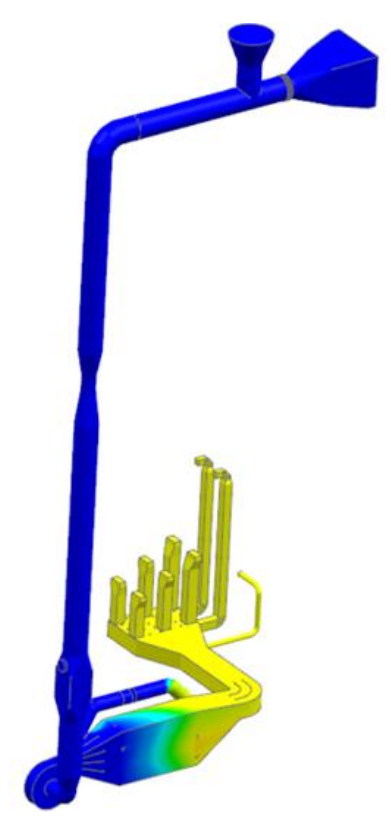

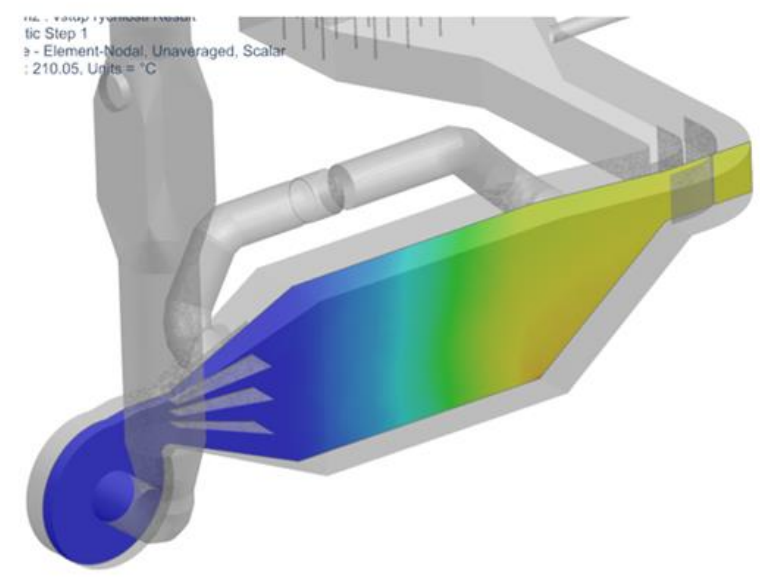

Fig. 13. Simulation of air temperature in ducts

Figure 14 shows the results of flow velocity in the pipeline. The highest medium velocity is seen at the top of the fan outlet. Figure 15 shows the course of temperature during start-up of incinerator.
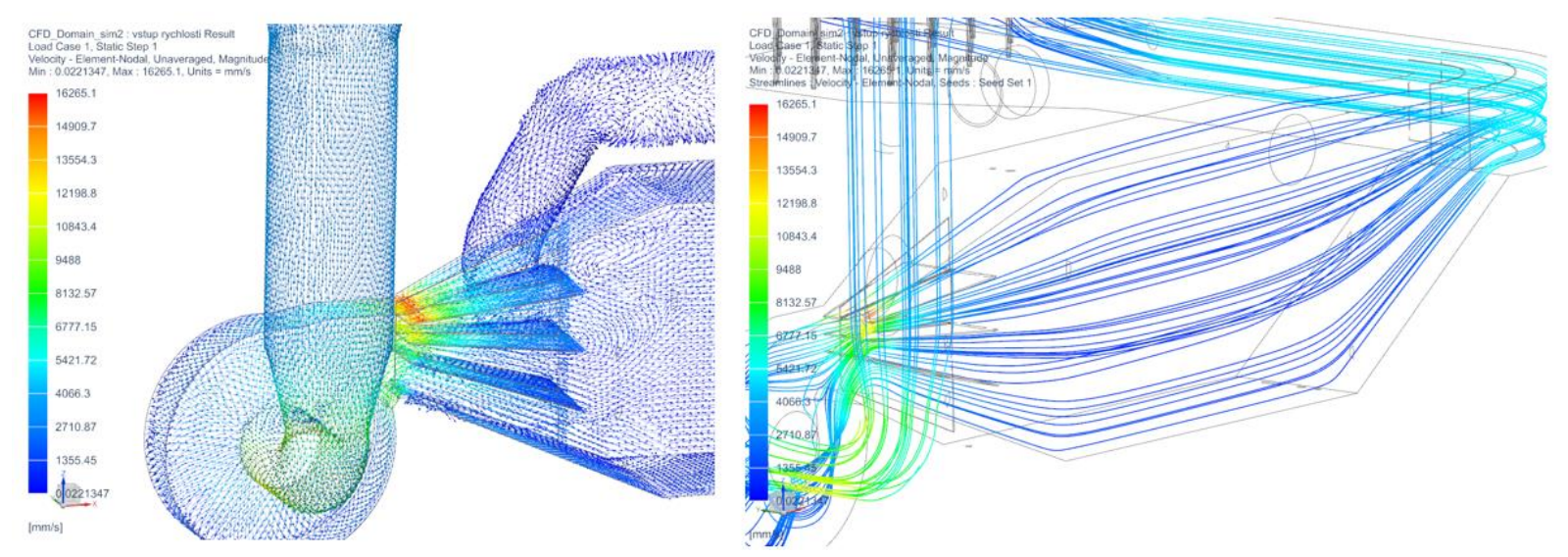

Fig. 14. Simulation of flow velocity in pipeline

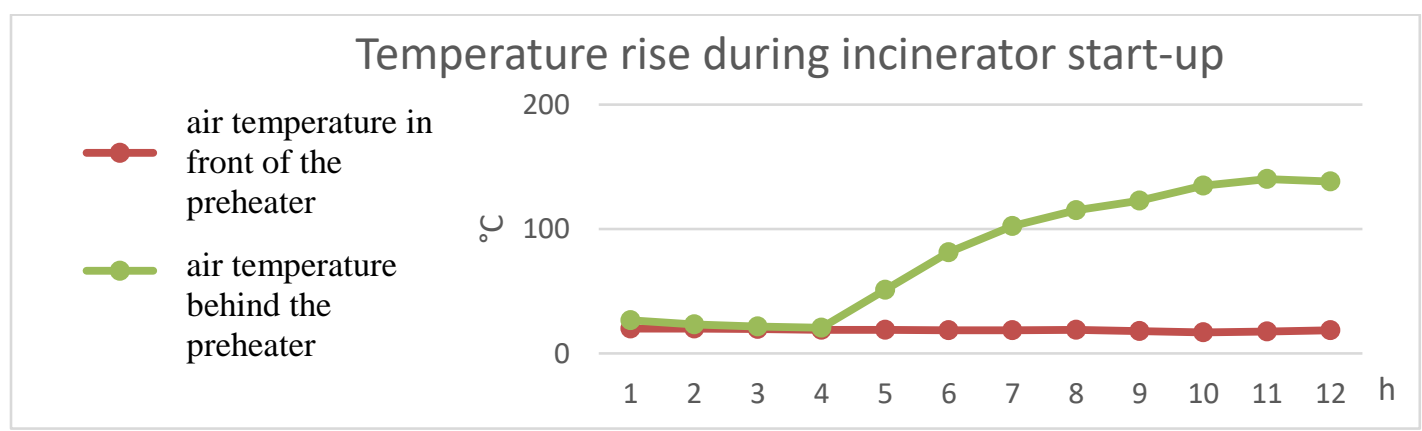

Fig. 15. Temperature course after preheating when the incinerator enters the operating mode 


\section{Conclusion}

Parameterized sub-models of individual parts of piping system and supports have been created, which can now be easily modified and folded into larger units. For models, bolts and other fasteners can be simply suppressed or replaced with logical parameters. The structure of the sub-models allows the flat pattern of the sheet metal parts for laser as well as the drawings of the individual parts to be automatically generated. Created parameterized models lead to time savings and thus also financial savings.

It is also planned that automated sub-models will be stacked one after another, without manual definition of constraints.

\section{Acknowledgments}

Authors of the work presented herein gratefully acknowledge the support in the form of the institutional funding for long-term strategic development of the University of West Bohemia provided by the Ministry of Education of the Czech Republic.

\section{References}

[1] Chval, Z.; Sedláček, F. \& Kemka, V. (2017). Usage of FEM simulations in design of piping systems. Manufacturing Technology, Vol.17, No. 4, pp. 469-473, ISSN: 1213-2489

[2] Marek, V \& Hajicek, Z (2017). Thermal Simulations Based on Macro-Models. Proceedings of the 28th DAAAM International Symposium, Zadar-Croatia, ISSN 1726-9679, ISBN 978-3-902734-11-2, Katalinic, B. (Ed.), pp.06270634, Published by DAAAM International, Vienna-Austria, DOI: 10.2507/28th.daaam.proceedings.089

[3] Marek, V. (2016). Basic Research of Thermal Transfer Simulations. Proceedings of the 27th DAAAM International Symposium, Zadar-Croatia, ISSN 1726-9679, ISBN 978-3-902734-08-2, Katalinic, B. (Ed.), pp.0578-0585, Published by DAAAM International, Vienna-Austria, DOI: 10.2507/27th.daaam.proceedings.085

[4] Marek, V. (2017). Vacuum technology for woodchips drying. 25th EUBCE. European Biomass Conference and Exhibition Proceedings. Stockholm. ISSN: 22825819. Vol. 17. pp.168-174

[5] Jenicek, S.; Kotesovec, V.; Kalina, T. \& Opatova K. (2018) Design of heating equipment for moulding organic polymer-based composites. Proceedings of the 28th DAAAM International Symposium, Zadar-Croatia, ISSN 17269679, ISBN 978-3-902734-11-2, Katalinic, B. (Ed.), pp.0273-0278, Published by DAAAM International, ViennaAustria, DOI: 10.2507/28th.daaam.proceedings.039

[6] Sedlacek, F. \& Lasova, V. (2018). Additive Manufacturing of PA6 with Short Carbon Fibre Reinforcement using Fused Deposition Modelling. 3rd International Conference on Composite Materials and Material Engineering, ICCMME 2018, Singapore, ISSN: 0255-5476, Vol. 928, pp. 26-31, DOI: 10.4028/www.scientific.net/MSF.928.26

[7] Sarkar D. (2015). Thermal Power Plant - Design and Operation. Imprint: Elsevier. p.612. ISBN: 9780128015759

[8] Konar, R.; Mician, M.; Patek, M. \& Kadas, D. (2016). Finite Element Modeling and Numerical Simulation of Welding at the Repair of Gas Pipelines with Steel Sleeve. Manufacturing Technology, Vol. 16, No.2, pp. 360-365, ISSN 1213-2489

[9] Zmindak, M.; Mesko, J.; Pelagic, Z. \& Zrak, A. (2014). Finite Element Analysis of Crack Growth in Pipe-lines. Manufacturing Technology, Vol. 14, No.1, pp. 116-122, ISSN 1213-2489

[10] Kusmierczak, S. (2015). Evaluation of Degradation of Heat Stressed Pipelines. Manufacturing Technology, Vol. 15, No.6, pp. 1006-1010, ISSN 1213-2489

[11] Lindapter. Online. 30.9.2019. www.lindapter.com/Products/Girder_Clamp/1/Type_B_Flat_Top 